\title{
Can ECAP Measures Be Used for Totally Objective Programming of Cochlear Implants?
}

\author{
Colette M. McKay, ${ }^{1,2}$ Kirpa Chandan, ${ }^{1}$ Idrick Akhoun, ${ }^{1}$ Catherine Siciliano, ${ }^{1}$ and Karolina Kluk ${ }^{1}$ \\ ${ }^{1}$ Audiology E Deafness Research Group, School of Psychological Sciences, The University of Manchester, Ellen Wilkinson Building, \\ Manchester, M13 9PL, UK \\ ${ }^{2}$ The Bionics Institute of Australia, 384-388 Albert St., East Melbourne, 3002, Australia
}

Received: 15 April 2013; Accepted: 6 September 2013; Online publication: 19 September 2013

\begin{abstract}
An experiment was conducted with eight cochlear implant subjects to investigate the feasibility of using electrically evoked compound action potential (ECAP) measures other than ECAP thresholds to predict the way that behavioral thresholds change with rate of stimulation, and hence, whether they can be used without combination with behavioral measures to determine program stimulus levels for cochlear implants. Loudness models indicate that two peripheral neural response characteristics contribute to the slope of the threshold versus rate function: the way that neural activity to each stimulus pulse decreases as rate increases and the slope of the neural response versus stimulus current function. ECAP measures related to these two characteristics were measured: the way that ECAP amplitude decreases with stimulus rate and the ECAP amplitude growth function, respectively. A loudness model (incorporating temporal integration and the two neural response characteristics) and regression analyses were used to evaluate whether the ECAP measures could predict the average slope of the behavioral threshold versus current function and whether individual variation in the measures could predict individual variation in the slope of the threshold function. The average change of behavioral threshold with increasing rate was well predicted by the model when using the average ECAP data. However, the individual variations in the slope of the thresholds versus rate
\end{abstract}

Correspondence to: Colette M. McKay - The Bionics Institute of Australia · 384-388 Albert St., East Melbourne, 3002, Australia. Telephone: +61-3-96677541; email: cmckay@bionicsinstitute.org functions were not well predicted by individual variations in ECAP data. It was concluded that these ECAP measures are not useful for fully objective programming, possibly because they do not accurately reflect the neural response characteristics assumed by the model, or are measured at current levels much higher than threshold currents.

Keywords: cochlear implant, thresholds, ECAP, loudness

\section{INTRODUCTION}

The introduction of reversed telemetry in cochlear implant (CI) systems made it possible to efficiently measure the response of the auditory nerve to electrical stimulus pulses and led to the expectation that this measure would facilitate a fully objective method to set the signal processor program parameters for individuals. However, many studies have now shown that the correlation between electrically evoked compound action potential (ECAP) thresholds and behavioral thresholds or comfortably loud levels is only moderate at best (Abbas et al. 1999; Hughes et al. 2000; Gordon et al. 2002, 2004a; Thai-Van et al. 2004; Cafarelli Dees et al. 2005; McKay et al. 2005; Potts et al. 2007) and deteriorates as the rate of stimulation used for behavioral measures increases. As modern implant systems use moderate to high rates of stimulation, the poor predictive power of ECAP thresholds prevents them from being used in isolation to set the program levels for each patient. Currently, the contour of ECAP thresholds across electrodes (or an amended version of this) 
together with one or more behavioral measurements is used in combination to set a preliminary program for infants and children (Smoorenburg et al. 2002; Gordon et al. 2004a, b). However, these ECAP-based maps have been shown by some researchers to be suboptimal for speech understanding (Seyle and Brown 2002; Smoorenburg et al. 2002). In this study, it was hypothesized that ECAP measures that characterize neural response properties in individuals for different rates of stimulation could be used to obtain a better prediction of high-rate behavioral thresholds than using ECAP thresholds alone.

McKay et al. (2005) discussed some possible reasons for the poor correlation between ECAP and behavioral thresholds. The standard ECAP amplitude (measured at a low probe rate of 40 or 80 pulses per second-pps) is related to the neural response to a single stimulus pulse in isolation. In contrast, the behavioral threshold for a pulse train is influenced by additional peripheral and central factors. At the periphery, the neural response to each pulse in a pulse train is influenced by the preceding pulses via refractory and adaptive mechanisms. The higher the pulse rate, the smaller the neural response to each pulse in the train (except the first pulse). The perceptual response to a pulse train is also influenced by central temporal integration. In a temporal integration model, the peripheral neural activity that occurs within an integration window of several milliseconds is integrated and the output of the integrator is used to make decisions about threshold, loudness, and intensity discrimination. McKay and McDermott (1998) and McKay et al. (2013) used such a model to investigate the effect of interpulse intervals on loudness. The data were fit with a model that combined neural adaptation effects with temporal integration. In that context, and in this paper, the term "adaption" is used to refer to the lowering in neural response magnitude due to increases in rate or stimulus duration regardless of the mechanisms underlying the change. In the above model, subjects who experience a high degree of adaptation are predicted to have flatter threshold versus rate functions than those with lesser degrees of adaptation, as, with an increase in rate of stimulation, the influence of temporal integration (more stimulus pulses occur in the integration window) would be counterbalanced by a decreasing neural response to each pulse. Another important factor that influences the slope of the threshold versus rate function is the slope of the neural response versus current function. The steeper the growth of neural activity with increase in current, the smaller the changes of current that are needed to maintain threshold or equal loudness as rate changes. The fact that equal loudness versus rate functions are generally steeper than threshold versus rate functions is consistent with the neural response versus current function becoming steeper at high stimulus levels (McKay et al. 2003).

It can be hypothesized, therefore, that differences among individuals of the slopes of the threshold (or equal loudness) versus rate functions are contributed to by differences in the degree of adaptation and the current to neural response slope among individuals. The standard ECAP threshold is usually highly correlated with (although higher than) single pulse or low-rate (e.g., 40 or $80 \mathrm{pps}$ ) behavioral thresholds (Brown et al. 1996, 1998; Zimmerling and Hochmair 2002), for which adaptation effects are minimal. It is evident then that the poorer correlation of ECAP thresholds with higher rate behavioral thresholds is due to individual variation in the slope of the behavioral threshold versus rate function. If that slope can also be predicted using alternative ECAP measures, then it should be possible to use it in combination with the prediction of the low-rate behavioral threshold to predict the higher rate thresholds. In this study, it was hypothesized that the slope of the behavioral threshold versus rate function would be correlated with measures of individual neural adaptation and/or the rate of growth of neural response with level, as estimated from ECAP measures.

\section{METHODS}

\section{Subjects}

Eleven post-lingually deaf adult users of cochlear implants participated in the experiment. All were users of the CI24RE implant and Freedom system manufactured by Cochlear Ltd, with the exception of one subject (S5) who had a CI24M implant. Three subjects (S1, S2, S8) were later removed from the study due to absent or poorly defined ECAPs. Table 1 shows details of the etiology and implant use for the remaining eight subjects.

\section{Procedures}

\section{Electrophysiological measures}

ECAP measurements were obtained for each subject via neural response telemetry (NRT) and Custom Sound Evoked Potential software provided by Cochlear Ltd. The biphasic stimulus pulses were presented in monopolar (MP1) mode and had a phase duration of $25 \mu$ s and interphase gap of $7 \mu$ s. The active electrode position was initially E12. In these implants, current is controlled in logarithmic steps called currents levels (CLs). Each CL step is equal to 0.18 or $0.16 \mathrm{~dB}$ for the CI24M and CI24RE implants, respectively. 


\begin{tabular}{|c|c|c|c|c|}
\hline \multicolumn{5}{|c|}{ TABLE 1} \\
\hline \multicolumn{5}{|c|}{ Subject details } \\
\hline Subject & Etiology & Duration of profound deafness (years) & Implanted ear & Duration of implant use (years) \\
\hline S3 & Familial progressive & 9 & $\mathrm{R}$ & 4 \\
\hline S4 & Head injury & 39 & $\mathrm{~L}$ & 4 \\
\hline S5 & CSOM & 14 & $\mathrm{R}$ & 10 \\
\hline S6 & Progressive & 38 & $\mathrm{~L}$ & 3 \\
\hline S7 & Progressive & 10 & $\mathrm{R}$ & 5 \\
\hline S9 & Congenital progressive & 22 & $\mathrm{~L}$ & 5 \\
\hline S10 & Viral infection & 9 & $\mathrm{R}$ & 5 \\
\hline S11 & Idiopathic sudden & 7 & $\mathrm{R}$ & 5 \\
\hline
\end{tabular}

First, an ECAP amplitude growth function (amplitude versus CL) was obtained using the standard forward masking procedure (Brown and Abbas 1990). This function was used initially to determine the presence of an ECAP and the CL range over which a good ECAP could be identified. If ECAPs were unsatisfactory on electrode E12, then electrodes E20 or E4 were tested. Three subjects (S1, S2, S8) were removed from the study at that point due to the absence of useful ECAPs on all three electrodes. In three other cases, useful ECAPs were found on electrodes other than E12, so the whole experiment (including behavioral measures) was performed using these alternative electrodes: E20 (S7, S10) and E4 (S5). The slope of the ECAP amplitude growth function (in decibel per decibel-denoted $S_{\mathrm{ECAP}}$ ) was calculated for later use in the loudness modeling, and the visual ECAP threshold (lowest CL at which a recognizable ECAP waveform was apparent) was noted for reader reference only.

Secondly, the amplitudes of ECAP evoked by a stimulus pulse when preceded by differing numbers and rates of masker pulses were measured. The amplitude measurements were undertaken for each of the first 20 pulses in each pulse train, and the pulse rates used were 500, 900, 1,200, 1,800, and 2,400 pps. In addition, the responses to three successive pulses that were approximately $40 \mathrm{~ms}$ after stimulus onset were recorded to assess the degree of adaptation for longer durations. Before starting this procedure, the maximum tolerable CL for the procedure was determined using masker pulse trains of 20 pulses at a rate of 2,400 pps. This CL was used for the first set of ECAP amplitude measurements.

Figure 1 shows a schematic diagram of the steps used to obtain the ECAP amplitude evoked by the $n$th pulse in a pulse train. In condition A, the artifact and response after $n$ pulses ( $n-1$ maskers and one probe) was obtained. The interpulse intervals were determined by the chosen rate, and all current levels in the pulse train were identical. Similarly, in condition B, the artifact and response after $n-1$ pulses was obtained. Subtracting B from A produced the artifact and response due to the $n$th pulse only. The artifact was then determined by subtracting condition $\mathrm{D}$ from condition $\mathrm{C}$. The masker pulse in conditions $\mathrm{C}$ and $\mathrm{D}$ was $10 \mathrm{CL}$ greater than the probe $\mathrm{CL}$ and preceded the probe in condition $\mathrm{C}$ by $500 \mu$ s. Finally, the response to the $n$th pulse was determined by subtracting the probe artifact from the responseplus-artifact obtained from conditions $\mathrm{A}$ and $\mathrm{B}$ (i.e., $[A-B]-[C-D])$. Before the runs of each rate (separated in time), the response to the first pulse (i.e. a single isolated pulse) was determined using the forward masking method described by Brown and Abbas (1990). The masker-probe interval and masker-probe CL offset were the same as shown for condition $\mathrm{C}$ in Figure 1. Thus, there were five or more measurements of ECAP amplitude for the first pulse (one for each rate under investigation and repetitions within a measurement run for each rate for some subjects, to check for response adaptation between conditions). These amplitudes were always similar and were averaged for later analysis.

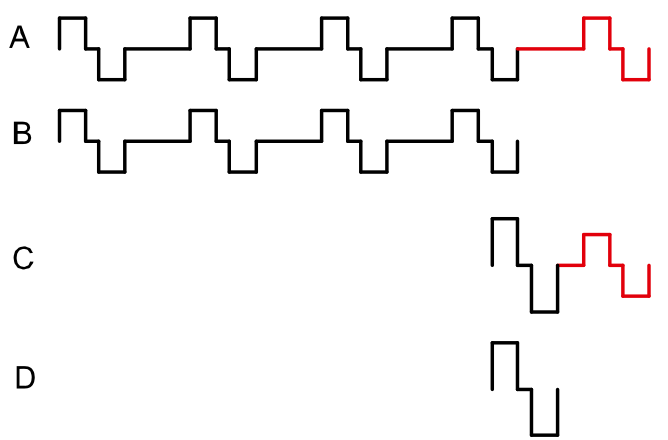

FIG. 1. A schematic diagram showing the four measurement buffers used to derive the ECAP waveform of the fifth pulse in a pulse train. Condition A includes five equal pulses at the test rate, four being maskers (black) and the last one being the probe pulse (red). Condition B includes only the four masker pulses of A. Subtraction of B from A gives the ECAP and artifact for the fifth pulse. Condition $\mathrm{C}$ contains the probe pulse preceded by a masker pulse that has a level $10 \mathrm{CL}$ higher than the probe pulse and a short interpulse interval of $500 \mu \mathrm{s}$ and $\mathrm{D}$ contains the masker pulse of $\mathrm{C}$ on its own. Subtraction of $D$ from $C$ gives the probe artifact, which can then be subtracted from the difference between $A$ and $B$ to obtain the artifact-free probe response to the fifth pulse. 
ECAP recordings were sampled at $20 \mathrm{kHz}$ and amplified by $50 \mathrm{~dB}$ for CI24RE implants and by $60 \mathrm{~dB}$ for the participant with a CI24M implant. The sampling delay was $122 \mu$ s for CI24RE implants and $68 \mu$ s for the CI24M implant. ECAPs were measured in $1,600 \mu$ s duration epochs. The probe rate varied with the masker rate and duration (maximum period of $1 \mathrm{~s}$ ) to ensure adequate neural recovery between measurement epochs. The ECAP amplitude for each masker condition (number and rate of masker pulses) was determined from the online average of 50 epochs. The recording electrode was two electrodes more apical than the stimulus electrode (except when E20 was the stimulus electrode, in which case E18 was the recording electrode) and used the MP2 electrode as the reference. Measurement data were analyzed offline using MatLab software to produce ECAP waveforms. The N1 and P1 peaks were visually determined and the ECAP amplitude was defined as the N1-P1 voltage difference. The noise floor was approximately $10 \mu \mathrm{V}$ in these recordings, and ECAP amplitudes below $10 \mu \mathrm{V}$ were considered to be noise.

The measure of adaptation $(A)$ for each masker rate was the average ECAP amplitude obtained from the third to the 20th pulse expressed as a fraction of the ECAP amplitude from the first pulse $\left(A m p_{1}\right)$.

$A=A m p_{1}^{-1}\left(1 / 18^{*} \sum_{3}^{20} A m p_{n}\right)$

An $A$ of 0 represents a total abolition of the ECAP response and an $A$ of 1 represents no reduction in ECAP amplitude relative to the first pulse.

Following the measurements at the maximum tolerable level, the same data were collected at lower stimulus current levels, provided that ECAP amplitudes remained significantly above the noise floor. Levels were chosen that spanned the range where ECAPs for a single pulse were clearly evident in the amplitude growth function.

\section{Behavioral measurements}

Detection thresholds were obtained using pulse trains of $500 \mathrm{~ms}$ duration. Pulse rates of 40, 250, 500, 900, 1,200, 1,800 , and 2,400 pps were used. Stimulation mode was MP1+2, pulse duration was $25 \mu \mathrm{s}$, and interphase gap was $7 \mu$ s. Note that the stimulus monopolar reference electrode $(M P 1+2)$ was slightly different to that for the ECAP measures (MP1). The two different reference electrodes are those typically used in each case, and the ECAP measures ideally require different reference electrodes (MP1 and MP2) to be used for stimulus and recording electrodes. Stimuli were controlled and subject responses recorded, using ImPResS software (developed at Melbourne University in collaboration with the MRC-CBU in Cambridge, UK). The software directly controlled the delivery of the electrical stimulus to the implanted electrodes via an interface using a SPEAR research processor (developed at the Hearing CRC in Melbourne). A response box was used to visually represent trial time intervals and to record subject responses.

Thresholds were obtained using an adaptive threeinterval three-alternative forced choice task, in which subjects were asked to nominate which of three intervals (separated by $500 \mathrm{~ms}$ ) in each trial contained a sound. The stimulus occurred in one randomly selected interval only. A two-down one-up adaptive procedure was used with a step size of $4 \mathrm{CL}$ until two reversals were obtained and then a step size of 2 CL until a total of 10 reversals were obtained. Threshold was calculated as the average CL at the final six reversals. After thresholds were obtained for each rate of stimulation, a second set of thresholds was obtained using the reverse order of testing to limit any effects of practice due to test order. If a repeated threshold was more than 6 CL different from the first measurement, a third threshold was measured. The two (or three) thresholds for each rate were averaged for further analyses.

\section{Temporal integration model of behavioral threshold}

The effect of rate on behavioral threshold has been modeled using a temporal integration model and has also been shown to successfully explain the effect of interpulse intervals on loudness in cochlear implantees (McKay and McDermott 1998) and to explain a variety of temporal effects, including those of rate, duration, and masker-probe interval, on thresholds in cochlear and auditory midbrain implantees (McKay et al. 2013). In this model, the slope of the behavioral threshold versus rate function depends on two key factors that vary between individuals. This slope is modeled to be flatter when the neural response to each pulse decreases more rapidly as rate increases and when neural activity increases faster with increases in stimulus current. The objective of this experiment was to evaluate whether these two model parameters can be inferred from ECAP measurements and, thus, to be able to predict the way that individual behavioral thresholds change with rate using only objective measurements. First, the average ECAP data was input into this temporal integration model to see if it could predict the slope of the average behavioral threshold versus rate function. Following this, regression analyses were performed to see whether individual variability in the ECAP measures could predict differences in behavioral threshold slopes among subjects.

In the model, the input to the temporal integration (TI) window is the neural response to each stimulus 
pulse. Thus, the way that the neural response to each pulse changes with rate of stimulation is an important model parameter in this experiment. In the present paper, the ECAP amplitudes for each subject were assumed to be proportional to the number of neurons that responded to the corresponding stimulus pulse. This is a first approximation only, since other factors such as synchrony will also affect the ECAP amplitude. The response to the first pulse in a pulse train was assigned a nominal value of 1 , and the responses to the second and subsequent pulses were assigned a value of the mean ECAP amplitude (across subjects) relative to that of the first pulse for that particular pulse and stimulus rate. Thus, all neural responses were denoted as a fraction of the response to the first pulse for any particular stimulus CL and rate.

The TI window used in this paper is the same as was successfully used by McKay et al. (2013) to explain a range of CI data and follows the form used by authors in the acoustic hearing field (Oxenham and Moore 1994; Oxenham 2001; Plack et al. 2002):

$$
\begin{aligned}
& W(t)=(1-w) \exp \left(t / T_{b 1}\right)+w \exp \left(t / T_{b 2}\right), \quad t<0 \\
& W(t)=\exp \left(-t / T_{a}\right), \quad t \geq 0,
\end{aligned}
$$

where $W(t)$ is the weight applied at time $t$ relative to the peak of the function, $T_{a}$ and $T_{b 1}$ together define the short time constant associated with temporal resolution, $T_{b 2}$ defines a longer tail of the window associated with forward masking and the effect of duration, and $w$ is the weighting of the long versus short time constants. Oxenham (2001) derived the integration window shape to best fit forward masking data in normal hearing listeners: the best-fitting values of the parameters were $T_{a}=3.5 \mathrm{~ms}, T_{b 1}=4.6 \mathrm{~ms}, T_{b 2}=16.6 \mathrm{~ms}$, and $w=0.17$. Since McKay and McDermott (1998) showed that TI windows fitted to CI data had similar widths to those of normal hearing subjects and did not vary significantly among CI subjects, it was assumed that the above parameters would be appropriate to use in the model here and that variations in the TI window did not contribute to variations in the effect of rate on threshold.

The output of the window was the weighted sum of all neural responses as per Eq. 2. A function of window output versus time was calculated by moving the peak of the window $(t=0)$ along the length of the stimulus. Detection threshold was assumed to be the stimulus level that evoked a fixed criterion maximum window output (assuming that internal noise was constant across experimental conditions-that is, a criterion signal-to-noise ratio).

In the final model step, to predict the average effect of rate of stimulation on behavioral threshold, the change in input stimulus level for each rate higher or lower than 500 pps that was required to achieve the same criterion window output as the 500 pps stimulus was calculated. This step required a function to relate stimulus current to neural response. The slope in decibel per decibel of the neural response versus current function, $S$, was first used as a free fitting parameter in the model. The way that $S$ changes with level can be hypothesized to be related to the way that loudness grows with level. McKay et al. (2003) showed that the slope of the loudness versus current function (on log-log scales) was fairly constant and similar across subjects for low currents (below the threshold of a 500-pps stimulus, approximately) and increased with level above a certain kneepoint current. Loudness can be plausibly assumed to be a power function of the temporal window output (summed neural response), since most decision criteria for changes in the output window necessary for detection of intensity changes can successfully be expressed as ratios (McKay et al. 2013). It follows that for threshold currents for rates of 500 pps and above, neural response is predicted to be a power function of current (a constant $S$, similar across subjects). In contrast, $S$ is predicted to increase at stimulus levels corresponding to threshold currents for rates lower than 500 pps and for suprathreshold stimulus levels, by an amount depending on where the subject-dependent kneepoint is situated. The fitted values of $S$ found by McKay and McDermott (1998) from psychophysical data ranged from 0.5 to 6.1 and significantly increased with level for the low-rate stimuli used in that experiment.

Although $S$ can theoretically be estimated using the ECAP growth function $\left(S_{\mathrm{ECAP}}\right)$, in practice, $S_{\mathrm{ECAP}}$ can only be measured using low-rate or single pulse stimuli, since the ECAP amplitudes are too small at low levels and high rates. $S_{\mathrm{ECAP}}$ was measured in this study using the standard forward masking procedure using a single pulse masker and, thus, was measured at stimulus levels above even the lowest rate threshold level. Thus, it was not expected that $S_{\mathrm{ECAP}}$ could be input into the model to predict behavioral threshold changes with increases in rate, since it was expected that $S$ would be level- and hence rate-dependent. Instead, $S$ was used as a free fitting parameter in the model, when using the average measured ECAP amplitudes as model input. The resultant fitted $S$ values and their changes with stimulus level were then compared to those previously obtained elsewhere using solely psychophysical data. $S_{\mathrm{ECAP}}$ is predicted to be greater than the fitted $S$ in the low-rate region and considerably greater than the fitted $S$ in the highrate region and is therefore not expected to be correlated with the slope of the threshold versus rate function above $500 \mathrm{pps}$. It may, however, be at least correlated with (although higher than) $S$ at 40 pps and may consequently be correlated with behavioral threshold differences between 40 and 250 pps.

In summary, the average ECAP amplitudes relative to that of the first pulse were input into the model which 
was then fit to the average behavioral threshold versus rate function using the $S$ parameter. The fitted $S$ values were compared to previously published values from psychophysical data at different stimulus levels. Next, the individual $A$ and $S_{\mathrm{ECAP}}$ measures were used in regression analyses to see if they could predict individual differences in the slope of the behavioral threshold versus rate function. It was hypothesized that differences in the way that $A$ changes with rate would predict differences in behavioral threshold slope in the highrate region (above $500 \mathrm{pps}$ ), where $S$ is predicted to be relatively constant and similar across subjects, and where adaptation effects would be likely to dominate. In contrast, it was hypothesized that $S_{\mathrm{ECAP}}$ would be correlated to the behavioral threshold slope in the very low-rate region (below $250 \mathrm{pps}$ ), where $S_{\mathrm{ECAP}}$ was measured, and where $A$ is assumed to be close to 1 .

\section{RESULTS}

\section{Behavioral measures}

Figure 2 shows the thresholds across rates for each subject. Panel A shows the thresholds in $\mathrm{dB}$ re $1 \mu \mathrm{A}$, while panel $\mathrm{B}$ shows the thresholds in decibel relative to the 500-pps threshold, replotted to more easily compare the slopes of the functions. Four sets of data (green symbols) are included in these graphs that are additional to the eight sets corresponding to the ECAP data sets, to illustrate the variation of the slopes across subjects and electrodes. It is clear from Figure 2 that the slopes vary more across subjects in the lowfrequency region $(<500 \mathrm{pps})$ than in the highfrequency region. The similarity of slopes above 500 pps across the subjects is illustrated by the small standard deviation of difference in threshold between 500 and 2,400 pps (mean difference $=5.8 \mathrm{~dB}, \mathrm{SD}=1.2 \mathrm{~dB}$, range 3.6 to 7.2 ). In contrast, the difference between thresholds at 40 and 500 pps although smaller on average varied more between subjects (mean difference $=3.7 \mathrm{~dB}$, $\mathrm{SD}=2.6 \mathrm{~dB}$, range from -0.1 to 7.9 ).

\section{ECAP measurements}

Figure 3 shows example ECAP waveforms obtained from S11 at the highest current level tested (210 CLs or $57.6 \mathrm{~dB}$ re $1 \mu \mathrm{A}$ ), with a masker of 2,400 pps and varying numbers of masker pulses. The thick black line is the ECAP obtained from a single pulse using the standard forward masking method. The thin colored lines are the ECAP waveforms from the third to the 19th pulse in the pulse train, obtained using the method illustrated in Figure 1, and the thick red line is the ECAP to the 20th pulse. The responses to the 2,400-pps pulse train showed an immediate reduction in amplitude relative to the first pulse, and an immediate

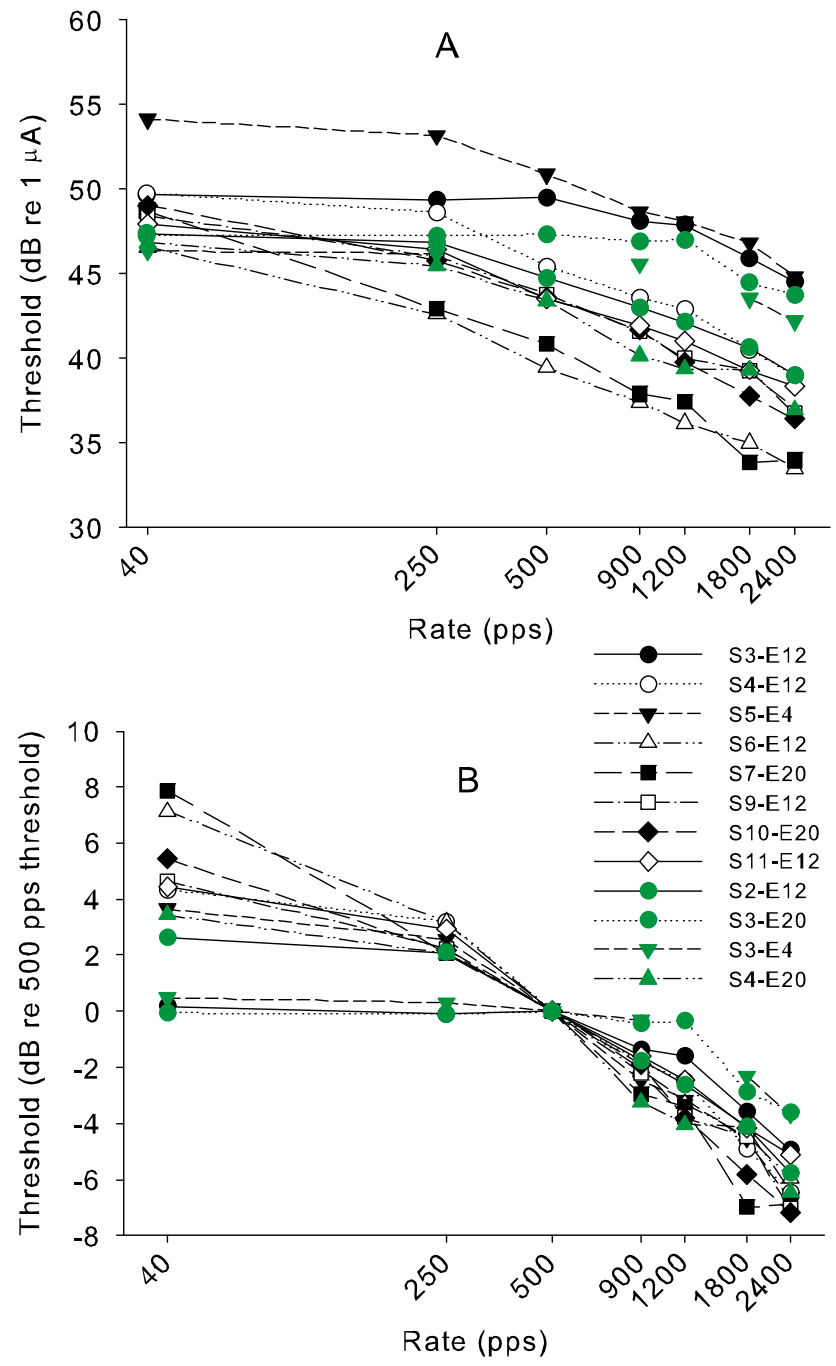

FIG. 2. A The behavioral thresholds of each subject in decibel re $1 \mathrm{~mA}$ as a function of stimulus rate. B The behavioral thresholds replotted in decibel re the 500-pps threshold. In both plots, the green data are additional behavioral data sets that were not used later for comparison with ECAP data (including a set for S2 who was later removed from the study due to inadequate ECAPs). The additional data are included to better illustrate the variability in slopes among subjects and electrodes.

increase in latency that did not vary much during the pulse train. The increase in latency after the first pulse was consistent across subjects when using high-rate maskers, and for some subjects, the latency alternated in step with the amplitude alternations. These changes in latency could possibly be associated with a change in response synchronicity if the first pulse induces a minimum post-stimulus response time in each activated fiber. If so, the ECAP amplitude variations do not necessarily reflect only changes in the number of neurons that responded, which was an assumption of the model. Another possible explanation for the change in morphology from the first to subsequent pulses is that there may be residual stimulus artifact. An assumption of 


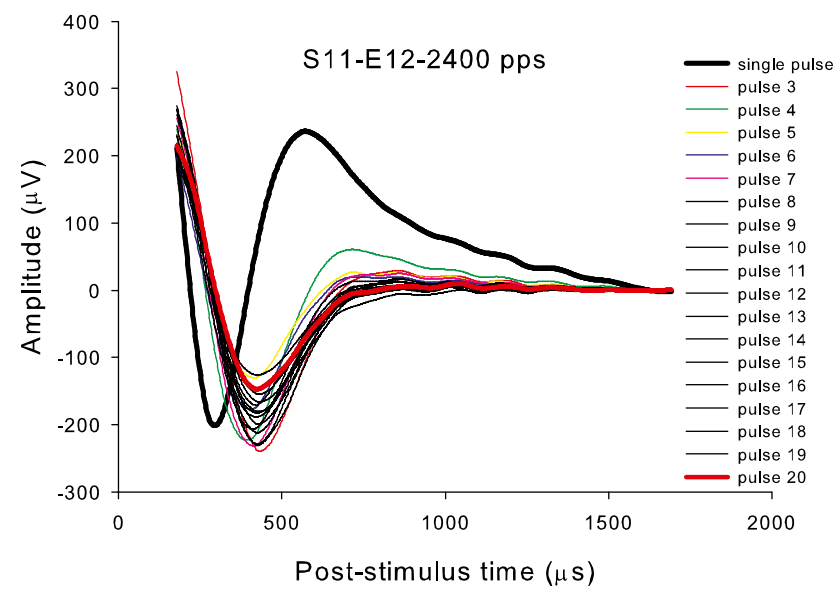

FIG. 3. An example set of ECAP waveforms derived for $\mathrm{S} 11$ using maskers of 2,400 pps and a stimulus level of $210 \mathrm{CLs}(57.6 \mathrm{~dB}$ re $1 \mathrm{~mA}$ ), showing the ECAP to the first pulse (thick black line) and the third to the 20th pulse (the last denoted by a thick red line).

the artifact rejection method is that the stimulus artifact of each pulse in the pulse train is identical to that of the first pulse. If this assumption is violated, then the morphology of the second and subsequent pulse responses may be distorted by residual artifact.

Figure 4 shows an example (S6) of the ECAP amplitudes for the first 20 pulses for different rates of stimulation at the highest level tested (190 CLs or $54.4 \mathrm{~dB}$ re $1 \mu \mathrm{A})$. In general, the amplitudes decreased with increasing pulse rate similarly in all subjects. Figure 5 shows the mean ECAP amplitudes relative to that of the first pulse (averaged across subjects) versus time after onset and includes the data collected around $40 \mathrm{~ms}$. The latter data were not collected for S4 as he was no longer available. It can be seen that the majority of the response reduction occurred over the first 20 pulses for each rate, with only small further reductions at the 40-ms duration.

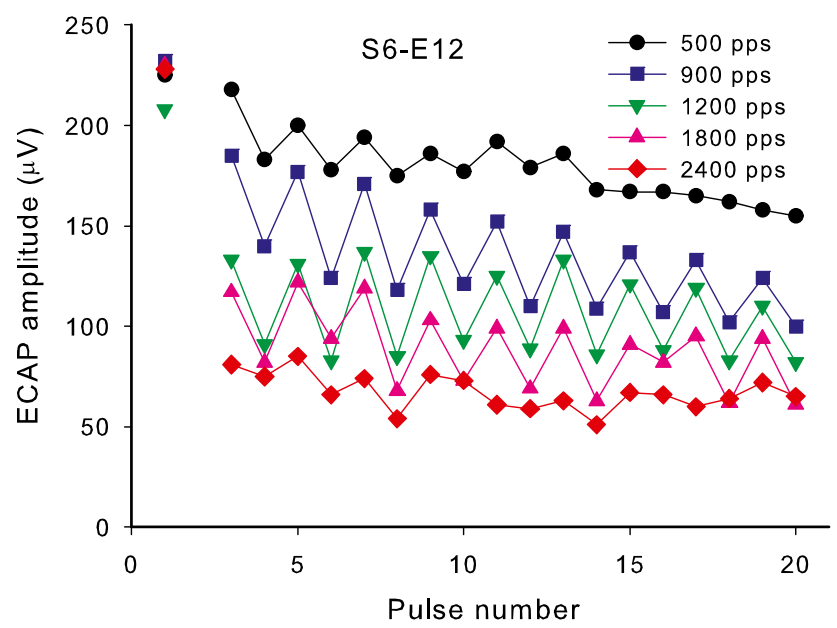

FIG. 4. A representative data set for $\mathrm{S6}$, showing ECAP amplitudes versus position of the probe pulse in the pulse train for different rates of stimulation and $190 \mathrm{CL}(54.4 \mathrm{~dB}$ re $1 \mathrm{~mA})$.

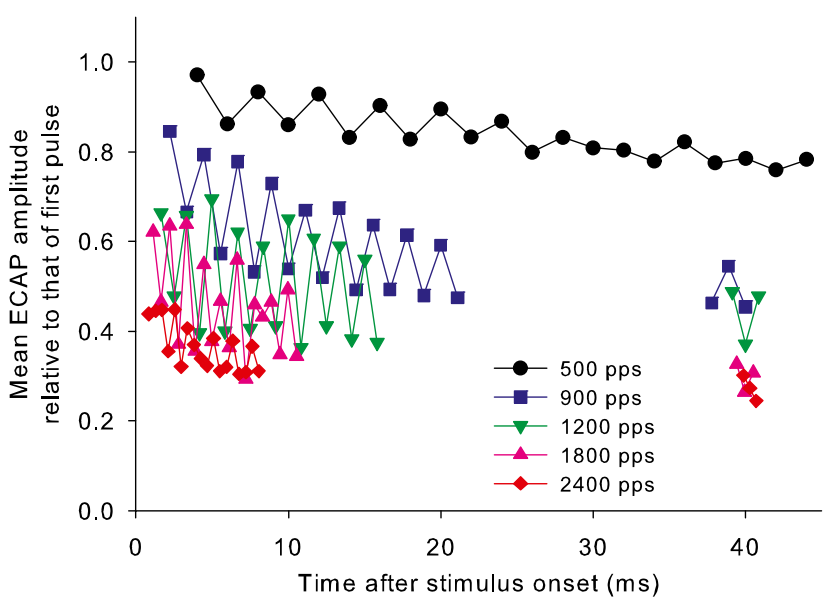

FIG. 5. Mean ECAP amplitudes normalized to that of the first pulse for each pulse between pulse 3 and pulse 20, and for three consecutive pulses near $40 \mathrm{~ms}$ duration. The amplitudes are shown versus time after masker onset. These normalized amplitudes were input into the loudness model (see text).

Figure 6 shows individual subject $A$ values and the means and standard deviations for different rates measured at the highest level. The mean data show a relatively linear decrease in average ECAP amplitude with logarithmic increments in rate above 500 pps.

Since the ECAP data were measured at current levels somewhat higher than those associated with behavioral thresholds, an important question is whether the $A$ values vary with stimulus level, and more importantly in the present experiment, whether values at high levels are correlated with values at low levels. Valid measures of $A$ could only be obtained at lower current levels in a subset (five) of subjects

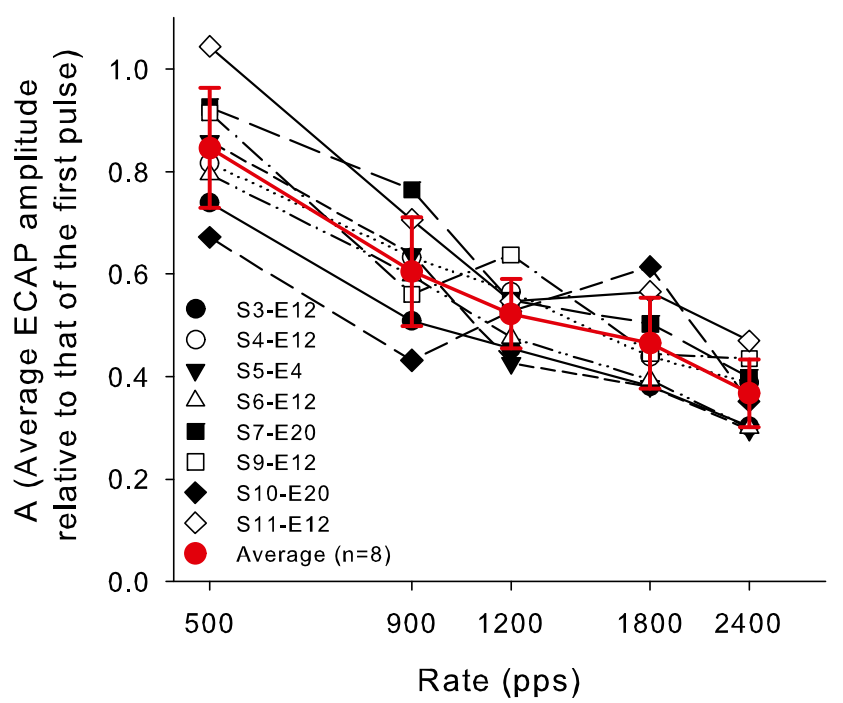

FIG. 6. Mean ECAP amplitudes for the third to the 20th pulse as a proportion of the amplitude to the first pulse (values of $A$ ) plotted against the masker rate. Individual subject data and mean data across subjects are shown. Error bars represent standard deviations. 
and mostly at the lower rates, due to the amplitudes being in the noise floor. Figure 7 shows the relation between values of $A$ obtained at the higher and lower levels. The regression shown in the figure shows that the $A$ values were significantly correlated across levels $\left(r_{(17)}^{2}=0.916, P<0.001\right)$ when combining data across subjects and electrodes and were greater on average (less reduction in amplitude) for the higher currents than for the lower currents by a constant amount of 0.045. A two-tailed paired $t$ test showed that this latter difference, although smaller compared to the differences between subjects and rates, was significant $\left(T_{17}=-3.127, P=0.006\right)$. The difference in CL between the higher and lower levels differed between subjects but was always in the range of 5-10 CL. It should be noted, however, that the current levels at which $A$ values could be obtained were higher than the high-rate behavioral thresholds. Therefore, when using the measured $A$ values to characterize individual adaptation, we are making an assumption that the values at behaviorally relevant levels are at least correlated with, and similar to, those at the measured levels for each subject.

For each subject, $S_{\mathrm{ECAP}}$ was derived from the slope in decibel per decibel of the ECAP amplitude growth function (with 40 pps measurement rate), fitted with a linear regression. $S_{\mathrm{ECAP}}$ values are shown in Table 2 , along with the ECAP threshold measured visually (V-NRT). The average $S_{\mathrm{ECAP}}$ was $4.1(\mathrm{~dB} / \mathrm{dB})$ with a standard deviation of $1.4 \mathrm{~dB} / \mathrm{dB}$.

\section{The loudness model: average data}

The average ECAP amplitudes (across subjects, measured at the higher level, as shown in Fig. 5) were used as input

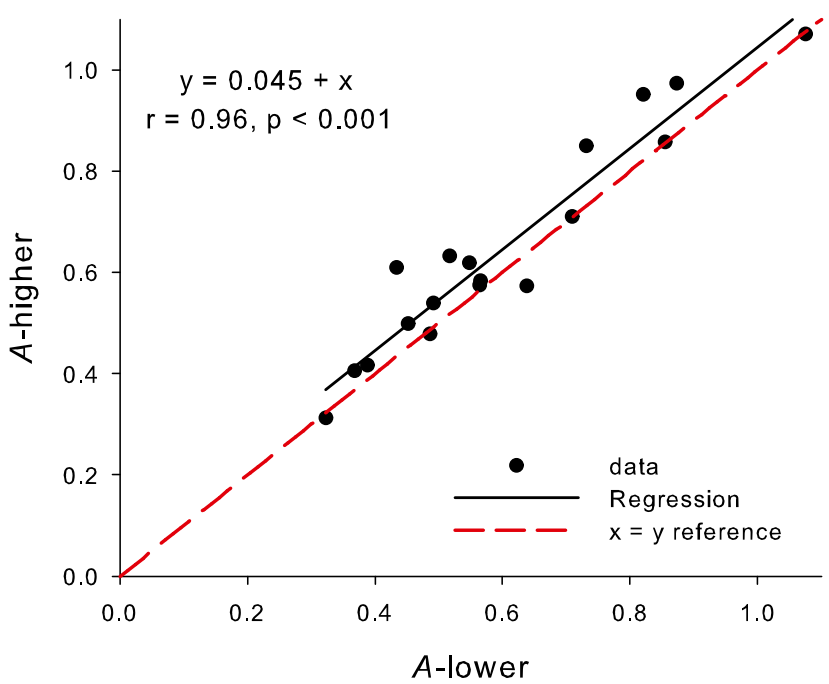

FIG. 7. Values of A measured at the higher stimulus level plotted against the value of $\mathrm{A}$ measured at the lower stimulus level for the same subject/rate. The black solid line is the regression line as per the equation, and the red dotted line is the equality line.

\begin{tabular}{|c|c|c|}
\hline \multicolumn{3}{|c|}{ TABLE 2} \\
\hline \multicolumn{3}{|c|}{$\begin{array}{l}\text { ECAP thresholds and slope of the ECAP growth function } \\
\qquad\left(S_{\text {ECAP }}\right) \text { in decibel per decibel }\end{array}$} \\
\hline Subject-electrode & $V$-TNRT $(C L$ and $d B$ re $1 \mu A)$ & $S_{E C A P}$ \\
\hline S3-E12 & $180,53.1$ & 3.3 \\
\hline S4-E12 & $185,53.9$ & 4.5 \\
\hline S5-E4 & $195,54.3$ & 2.5 \\
\hline S6-E12 & $155,49.2$ & 4.6 \\
\hline S7-E20 & $175,52.3$ & 2.0 \\
\hline S9-E12 & $160,50.0$ & 6.5 \\
\hline S10-E20 & $175,52.3$ & 4.5 \\
\hline S11-E12 & $175,52.3$ & 5.1 \\
\hline
\end{tabular}

to the loudness model, while using $S$ as a free fitting parameter, to assess whether the model could explain the average slope of the behavioral threshold versus rate data.

For each rate, the value of 1 was applied to the neural response to the first pulse and a value equal to the mean relative ECAP amplitude (Fig. 5) for each of the responses to pulses 3 to 20. Since the response to the second pulse was not measured for technical reasons, its response was input as equal to the subsequent even numbered pulse (pulse 4). The amplitudes for pulse 21 and subsequent pulses were assigned to the average amplitude of the three pulses at $40 \mathrm{~ms}$ duration for each rate above 500 pps. The possibility of further adaptation after $40 \mathrm{~ms}$ was ignored since the TI window output had generally reached its maximum by $40 \mathrm{~ms}$, and the similar data of Hay-McCutcheon et al. (2005) was consistent with the present data in showing very little adaption after the 20th pulse. The TI window maximum output (across the $500 \mathrm{~ms}$ ) was then calculated for each rate and converted to a decibel scale. Finally, the fitting constant, $S$, the slope of the neural response versus current function, was adjusted so that the current adjustment needed to maintain a constant criterion TI window output for different rates was equal to the current adjustment in the behavioral data. Since the variation in behavioral threshold versus rate functions across subjects differed in the range above and below $500 \mathrm{pps}$, and the behavior of the fitting constant, $S$, was also predicted to differ in the range above and below 500 pps, the TI window output at 500 pps was used as the criterion output for adjustment of currents above and below this rate.

Figure 8 shows the average behavioral thresholds (filled circles) relative to the 500-pps threshold, together with the fitted model (open symbols). For 500 pps and above, a constant value of $S$ (1.55-red triangles) produced a very good fit to the data. However, this $S$ value did not predict the 40- and 250-pps thresholds. To correctly fit the change from 500 to 250 pps thresholds, a higher $S$ value of 2.68 was needed (blue triangle), and to fit the change in 


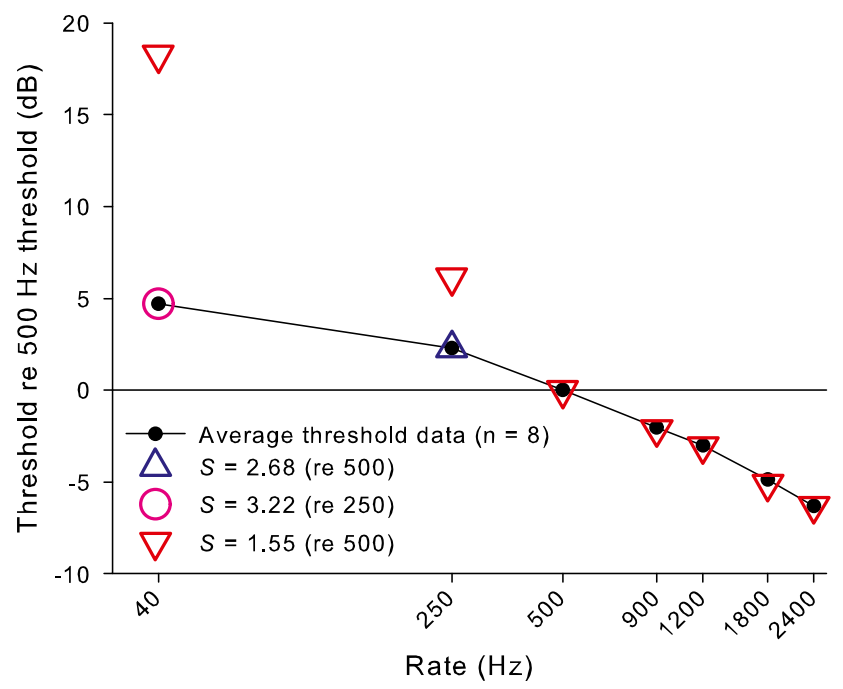

FIG. 8. Mean behavioral thresholds (solid circles and line) and model fits to the data (colored open symbols) for different rates plotted as a function of the 500-pps threshold. The open symbols are model fits to the data using the measured values of $A$ and using $S$ as a fitting parameter. A constant $S$ of 1.55 (red downward triangles) produces an excellent fit for all data points above 500 pps, but fails to predict the shallower slope below 500 pps. Increasing values of $S$ at higher levels (blue upward triangle and pink circle) are needed to fit the behavioral data.

threshold from 250 to $40 \mathrm{pps}$, an $S$ value of 3.22 was needed (pink triangle). Of course, it is possible to exactly fit the threshold at each rate by independently adjusting $S$ for each point. However, the fitted $S$ values are very similar to the average values found using a different set of subjects by McKay and McDermott (1998) using solely psychophysical data of a different nature (average $S$ of 1.45 at threshold and 3.3 at higher levels). Furthermore, the behavior of the $S$ values at different rates (constant at threshold for rates above 500 pps and increasing as rate decreases (or level increases) below $500 \mathrm{pps}$ ) matches the predicted behavior of $S$ based on loudness growth with level (McKay et al. 2003). Alternatively, to produce a model output using a constant $S$ that matches the much shallower slope below 500 pps compared to above $500 \mathrm{pps}$, an amplitude versus rate function that drops more steeply with rate at very low rates compared to higher rates would be required. This would be inconsistent with the ECAP amplitude data (Figs. 5 and 6) and also inconsistent with what is known physiologically.

As mentioned above, the measured $S_{\mathrm{ECAP}}$ was expected to be somewhat higher than the true $S$ at behavioral threshold at $40 \mathrm{pps}$. The average $S_{\mathrm{ECAP}}$ of $4.1 \mathrm{~dB} / \mathrm{dB}$ was indeed somewhat higher than the value (3.22) fit by the model to the average change in behavioral threshold between 250 and 40 pps and is broadly consistent with the value expected by an extrapolation of the fitted $S$ values to the higher current level at which the ECAP was measured. This consistency lends support for the general validity of the loudness model.

\section{Analysis of individual variability}

Since the behavior of both the psychophysical and electrophysiological data differed in nature above and below 500 pps, the ECAP predictors of the slopes of the behavioral thresholds versus rate functions were separately investigated in the two different rate ranges.

Above 500 pps, the behavioral slopes show only a small variability across subjects. According to the model, this variability could be due either to individual differences in $S$, or differences in the slope of the $A$ versus rate function. The former cannot be measured electrophysiologically, since the neural response is not measurable via the usual NRT measurements at the low stimulus currents needed for high-rate thresholds. However, it can be hypothesized that a steeper $A$ versus rate function will result in a flatter behavioral threshold versus rate function. To test this hypothesis, a regression analysis was performed to assess whether the difference between 500 and 2,400 pps behavioral thresholds (in decibels) could be predicted by the difference in $A$ between 500 and 2,400 pps (in dB: $20 \log (A 500 / A 2,400))$.

Although the trend in the relationship was in the hypothesized direction, it did not account for a significant amount of the variability in the threshold versus rate slope $\left(r^{2}(7)=0.07, P=0.53\right)$. This may be because there is very little variation between subjects in the slopes of $A$ or threshold between 500 and 2,400 pps (leading to poor statistical power), or because other factors (such as differences in $S$ across subjects) have more influence on behavioral threshold slopes than the slope of the $A$ versus rate function.

For the rate range below $500 \mathrm{pps}$, it is highly likely that the variation in $S$ across subjects and how it increases with level contribute significantly to the variation in slope of the threshold versus rate functions. Between 40 and $250 \mathrm{pps}$, for example, it is not expected that any significant reduction in $A$ would occur. The hypothesis was tested that higher $S_{\mathrm{ECAP}}$ values would be associated with flatter behavioral threshold slopes between 40 and $250 \mathrm{pps}$ (assuming that $S_{\mathrm{ECAP}}$ is at least correlated with the appropriate $S$ at the 40-pps behavioral threshold level). Again, although the regression showed a trend in the hypothesized direction, it did not account for a significant amount of the variability $\left(r_{(7)}^{2}=0.04, P=0.65\right)$, indicating that $S_{\mathrm{ECAP}}$ may not be a good predictor of $S$ at the appropriate lower level, as discussed above.

A third hypothesis was tested that a larger $A$ at $500 \mathrm{pps}$ (expressed as $20 \log (A 500))$ ) is associated with a greater difference between 40 and 500 pps thresholds. Once again, the relationship had a trend in the hypothesized direction but did not account for a significant amount of variance $\left(r_{(7)}^{2}=0.04, P=0.61\right)$. 


\section{DISCUSSION}

The present experiment attempted to find parameters of ECAP measurements that would predict the way that behavioral thresholds change with rate of stimulation. If successful, the prediction of the slope of the threshold versus rate function, along with the high correlations found between ECAP thresholds and low-rate behavioral thresholds, would allow highrate behavioral thresholds to be predicted using solely objective ECAP measures.

The average way that the amplitudes of ECAP declined with increasing rate of stimulation was input into a loudness model that successfully predicted the average slope of the threshold versus rate function, given plausible values of the slope of current versus excitation function that were consistent with previous models and the direct measurement of ECAP amplitude growth functions. However, the variability between individuals of the behavioral threshold versus rate slope was not well predicted by variability in individual ECAP measures. Importantly, the hypothesis that differences in the effect of rate on ECAP amplitude (or the effect of neural adaptation) would explain variation in the slope of the behavioral threshold versus rate function was not supported: for rates above 500 pps, both these slopes showed little variation between subjects, whereas for rates below 500 pps, there was little evidence of neural adaptation in spite of large variations in behavioral slopes between subjects. The consistency of fitted $S$ values when using the ECAP average amplitudes as model input with the previously published $S$ values derived from psychophysical data only, supports the idea that ECAP amplitudes generally reflect neural response amplitudes, although it is possible that ECAP amplitudes measured at high rates could be underestimates: the shift in response latency after the first pulse in a pulse train (see Fig. 3) most likely reflects increased neural response jitter that could both increase the latency and decrease the amplitude of the response averaged across the stimulus epochs. Thus, the ECAP amplitude may not reflect the number of neurons that were activated by the pulse and, hence, may underestimate the predicted loudness from the model.

Hay-McCutcheon et al. (2005) also measured individual differences in neural adaptation via ECAPs measured using a 1,000-pps pulse train and tested a hypothesis that their measure (analogous to the normalized amplitudes at $40 \mathrm{~ms}$ duration in the current data) would be correlated with the perceptual effect of stimulus duration on loudness and thresholds. The fact that no significant correlation was found is consistent with the current data, but may be also explained in part by the lack of consideration of differences in current-to-excitation slopes among the subjects in that experiment. If these slopes varied more than the adaptation effects for a 1,000-pps stimulus, it would lead to the observed lack of correlation in that experiment.

A conclusion from the current data is that the poor correlations between ECAP thresholds and high-rate behavioral thresholds that are documented in many published reports have been caused in major part by individual variation in how low rates affect behavioral thresholds, since this is the region where the majority of variation in the behavioral data is seen. Since neural adaptation does not seem to be a major factor at these low rates, alternative mechanisms must contribute to the variation in behavioral threshold slopes.

The loudness model suggested that a potentially important factor influencing the low-rate behavioral threshold slopes is the slope of the neural activity versus current function. If activity grows faster in one subject as current is increased, then the behavioral thresholds for different rates will be closer (that is the threshold versus rate function will be flatter) than for a different subject with slower activity growth with level. The higher currents associated with low-rate thresholds, as well as the steeper slope of the activity versus current function at higher currents, contribute to the generally flatter threshold function at low rates compared to high rates (McKay et al. 2013). Therefore, it is plausible that differences between subjects in low-rate threshold versus rate slopes may be largely due to differences in the slope of activity growth with current at high current levels. However, a measure of ECAP amplitude growth $\left(S_{\mathrm{ECAP}}\right)$ did not account for a significant amount variation of the behavioral threshold slopes in the low-frequency region. The predictive failure of this objective measure could be due to multiple factors: the model may be incorrect, although it is unclear what alternative parameters might be influencing the effect of rate on low-rate thresholds, or the fact that $S_{\mathrm{ECAP}}$ must be measured at suprathreshold levels may render it a poor estimate of $S$ at perceptual threshold.

The slope of the activity growth with current may be influenced by which neural elements are being activated. McKay et al. (2003) hypothesized that the steep increase in loudness above a certain kneepoint current in each individual may be due to activation of more central and tightly packed neural material in the internal auditory meatus. In subjects with poor spiral ganglion cell survival, it will be necessary to increase the current to higher levels to recruit sufficient activity for comfortable loudness, thus making it likely that these subjects will have a kneepoint in loudness growth at a point lower in their overall dynamic range than those with good spiral ganglion cell survival. Thus, subjects with poorer neural survival may have flatter threshold versus rate functions, particularly in the low-rate region, than those with better neural survival. 
The effect of cochlear health on pulse train thresholds has been studied in guinea pigs with cochlear implants (Middlebrooks 2004; Kang et al. 2010; Pfingst et al. 2011). In general, the findings showed that animals with deafened cochleae (absence of hair cells and dendrites and reduced spiral ganglion cell [SGC] density) generally had higher thresholds to pulse trains and the slopes of the threshold versus rate functions below 1,000 pps were flatter compared with animals that had remaining cochlear function. This animal data support the proposal that the flatter threshold versus rate slope for rates below 1,000 pps in some animals was due to activation of more central and tightly packed neural material in the internal auditory meatus in animals with poor SGC survival. Both the animal and human CI studies show an imperfect relationship, between absolute low-rate behavioral threshold and slope of the threshold versus rate function (Middlebrooks 2004; Bonnet et al. 2012; Zhou et al. 2012), and this can be attributed (particularly in humans) to the additional influence on thresholds of the distance of the electrode from the spiral ganglion or internal auditory meatus. Thus, the ECAP threshold itself is not a reliable objective correlate of the slope of the threshold versus rate function in the low-rate region. Instead, an objective measure that is highly correlated with spiral ganglion cell survival may help to predict the low-rate slope of the threshold versus rate function. One ECAPdependent measure that has been correlated with spiral ganglion cell survival in guinea pigs is the effect of pulse duration or interphase gap on the ECAP growth function (Prado-Guitierrez et al. 2006). Animals with better survival showed more shift in the ECAP amplitude growth functions (change in current for equal ECAP amplitude) when pulse timing parameters were changed than animals with poor survival. Thus, we could hypothesize that human subjects who show greater influence of pulse timing parameters on their ECAP amplitude growth functions (better neural survival) would have a steeper behavioral threshold versus rate function in the low-rate region. It remains to be tested whether this correlation exists, and, if so, whether it would allow prediction of high-rate behavioral thresholds with sufficient accuracy for completely objective cochlear implant fitting.

\section{CONCLUSIONS}

The variation between individuals in the way that ECAP amplitude changed when preceded by masker pulse trains of different rates did not predict the variation in the way that behavioral thresholds changed with rate and, thus, did not assist in improving the predictability of behavioral thresholds from ECAP measurements alone. A majority of the variation in the behavioral threshold versus rate slope occurred in the low rates $(<500$ pps $)$ where there was little effect of masker rate on
ECAP amplitude, leading to the proposition that the low correlations between ECAP thresholds and high-rate behavioral thresholds are instead due to individual differences in how neural activity grows with current, the latter mostly affecting the behavioral slope in the lowrate region. However, ECAP amplitude growth function slopes (objective correlates of neural activity growth) were not correlated with behavioral threshold versus current slopes in the low-rate region, probably due to the suprathreshold currents needed for the ECAP measures.

All regressions that estimated the influence of individual differences in the degree of adaptation or differences in neural activity versus current slope on the way that behavioral thresholds changed with rate provided relationships with a trend in the hypothesized direction, suggesting that statistically significant correlations might be found in an experiment with higher power. However, even if this were so, it is unlikely that the ECAP measures would provide sufficient predictive power to be used in individual objective programming, given the low $r$ values found here.

Taken together, the findings suggest that it is unlikely that ECAP measures additional to ECAP threshold can be combined with ECAP thresholds to predict high-rate behavioral thresholds with sufficient accuracy to eliminate the need for behavioral measures when fitting a cochlear implant.

\section{ACKNOWLEDGMENTS}

This study was funded by Cochlear Europe as a $\mathrm{PhD}$ studentship to the second author and by a project grant from the UK Medical Research Council to the first author. The authors thank the cochlear implant users who generously gave their time for this study. The Bionics Institute acknowledges the support it receives from the Victorian Government through its Operational Infrastructure Support Program.

Open Access This article is distributed under the terms of the Creative Commons Attribution License which permits any use, distribution, and reproduction in any medium, provided the original author(s) and the source are credited.

\section{REFERENCES}

Abbas PJ, Brown CJ, Shallop JK, Firszt JB, Hughes ML, Hong SH, STALlER SJ (1999) Summary of results using the nucleus CI24M implant to record the electrically evoked compound action potential. Ear Hear 20:45-59

Bonnet RM, Boermans PP, Avenarius OF, Briaire JJ, Frijns JH (2012) Effects of pulse width, pulse rate and paired electrode stimulation on psychophysical measures of dynamic range and speech recognition in cochlear implants. Ear Hear 33:489-496

Brown CJ, AbBas PJ (1990) Electrically evoked whole-nerve action potentials: parametric data from the cat. J Acoust Soc Am $88: 2205-2210$

Brown CJ, AbBas PJ, Borland J, Bertschy MR (1996) Electrically evoked whole nerve action potentials in Ineraid cochlear implant users: responses to different stimulating electrode configurations 
and comparison to psychophysical responses. J Speech Hear Res 39:453-467

Brown CJ, Abbas PJ, Gantz BJ (1998) Preliminary experience with neural response telemetry in the nucleus CI24M cochlear implant. Am J Otol 19:320-327

Cafarelli Dees D Et al (2005) Normative findings of electrically evoked compound action potential measurements using the neural response telemetry of the Nucleus CI24M cochlear implant system. Audiol Neurootol 10:105-116

Gordon KA, Ebinger KA, Gilden JE, Shapiro WH (2002) Neural response telemetry in 12- to 24-month-old children. Ann Otol Rhinol Laryngol 189:42-48

Gordon K, PAPsin BC, Harrison RV (2004A) Programming cochlear implant stimulation levels in infants and children with a combination of objective measures. Int J Audiol 43(Suppl 1):S28-S32

Gordon KA, Papsin BC, Harrison RV (2004в) Toward a battery of behavioral and objective measures to achieve optimal cochlear implant stimulation levels in children. Ear Hear 25:447-463

Hay-McCutcheon MJ, Brown CJ, AbBas PJ (2005) An analysis of the impact of auditory-nerve adaptation on behavioral measures of temporal integration in cochlear implant recipients. J Acoust Soc Am 118:2444-2457

Hughes ML, Brown CJ, Abbas PJ, Wolaver AA, Gervais JP (2000) Comparison of EAP thresholds with MAP levels in the nucleus 24 cochlear implant: data from children. Ear Hear 21:164-174

Kang SY, Colesa DJ, Swiderski DL, Su GL, Raphael Y, Pfingst BE (2010) Effects of hearing preservation on psychophysical responses to cochlear implant stimulation. J Assoc Res Otolaryngol 11:245-265

McKay CM, McDermott HJ (1998) Loudness perception with pulsatile electrical stimulation: the effect of interpulse intervals. J Acoust Soc Am 104:1061-1074

McKay CM, Henshall KR, Farrell RJ, McDermott HJ (2003) A practical method of predicting the loudness of complex electrical stimuli. J Acoust Soc Am 113:2054-2063

McKay CM, Fewster L, Dawson P (2005) A different approach to using neural response telemetry for automated cochlear implant processor programming. Ear Hear 26:38S-44S

McKay CM, Lim HH, Lenarz T (2013) Temporal processing in the auditory system: insights from cochlear and auditory midbrain implantees. J Assoc Res Otolaryngol 14:103-124
Middlebrooks JC (2004) Effects of cochlear-implant pulse rate and inter-channel timing on channel interactions and thresholds. J Acoust Soc Am 116:452-468

Oxenham AJ (2001) Forward masking: adaptation or integration? J Acoust Soc Am 109:732-741

Oxenham AJ, Moore BC (1994) Modeling the additivity of nonsimultaneous masking. Hear Res 80:105-118

Pfingst Be, Colesa dJ, Hembrador S, Kang SY, Middlebrooks JC, Raphael Y, Su GL (2011) Detection of pulse trains in the electrically stimulated cochlea: effects of cochlear health. J Acoust Soc Am 130:3954-3968

Plack CJ, Oxenham AJ, Drga V (2002) Linear and nonlinear processes in temporal masking. Acta Acust United Acust 88:348-358

Potts LG, Skinner MW, Gotter BD, Strube MJ, Brenner CA (2007) Relation between neural response telemetry thresholds, $\mathrm{T}$ - and C-levels, and loudness judgments in 12 adult nucleus 24 cochlear implant recipients. Ear Hear 28:495-511

Prado-Guitierrez P, Fewster LM, Heasman JM, McKay CM, Shepherd RK (2006) Effect of interphase gap and pulse duration on electrically evoked potentials is correlated with auditory nerve survival. Hear Res 215:47-55

SeYle K, Brown CJ (2002) Speech perception using maps based on neural response telemetry measures. Ear Hear 23:72S-79S

Smoorenburg GF, Willeboer C, van Dijk JE (2002) Speech perception in nucleus CI24M cochlear implant users with processor settings based on electrically evoked compound action potential thresholds. Audiol Neuro-Otol 7:335-347

Thai-Van H, Truy E, Charasse B, Boutitie F, Chanal JM, Cochard N, Piron JP, Ribas S, Deguine O, Fraysse B, Mondain M, Uziel A, Collet L (2004) Modeling the relationship between psychophysical perception and electrically evoked compound action potential threshold in young cochlear implant recipients: clinical implications for implant fitting. Clin Neurophysiol 115:2811-2824

Zhou N, Xu L, Pfingst BE (2012) Characteristics of detection thresholds and maximum comfortable loudness levels as a function of pulse rate in human cochlear implant users. Hear Res 284:25-32

ZimmerLing MJ, HochmaIR ES (2002) EAP recordings in Ineraid patients-correlations with psychophysical measures and possible implications for patient fitting. Ear Hear 23:81-91 